\title{
PROPOSTA DE SISTEMATIZAÇÃO DO ESTUDO ELASTOGRÁFICO DE LESÕES MAMÁRIAS PELA ULTRASSONOGRAFIA
}

Eduardo de Faria Castro Fleury*1, Jose Carlos Vendramini Fleury², Vilmar Marques de Oliveira ${ }^{3}$, Jose Francisco Rinaldi ${ }^{4}$, Sebastiao Piato 5 , Decio Roveda Junior ${ }^{6}$

Trabalho realizado na Santa Casa de Misericórdia de São Paulo, S. Paulo, SP

*Correspondência:

Santa Casa de

Misericórdia de São Paulo

Alameda Ministro Rocha

Azevedo, 1368, ap. 52

São Paulo - SP

CEP 01410-002

edufleury@ hotmail.com

\begin{abstract}
RESUMO
ОвJetivo. Propor uma sistematização do estudo elastográfico para emprego na rotina ultrassonográfica.

Métodos: Avaliação de 308 pacientes encaminhadas ao serviço de intervenção mamária do CTC-Gênese no período de 1 de maio de 2007 a 1 de março de 2008 para a realização de biópsia mamária percutânea. Antes da realização da biópsia percutânea foi realizado o estudo ultrassonográfico e a elastografia. As lesões foram primeiramente analisadas e classificadas conforme o léxico Bi-Rads pelo ultrassom convencional (modo B). Posteriormente, a elastografia foi realizada e analisada conforme sistematização proposta pelos autores, por meio das imagens obtidas durante a compressão e após a descompressão da área de interesse. As lesões foram classificadas conforme escores criados pelos autores: os escores 1 e 2 foram considerados benignos, o escore 3 como provavelmente benigno e 0 4 como suspeito para malignidade. Foram comparados os resultados obtidos entre os dois métodos com os resultados histológicos utilizando as áreas dentro das curvas ROC (curvas operadores dependentes). Resultados. A área dentro da curva para a elastografia foi de 0.952 com intervalo de confidência entre 0.910 e 0.966 , erro de 0.023 , e de 0.867 com intervalo de confidência entre 0.823 e 0.903 , erro de 0.0333 , para o ultrassom. Quando comparadas as áreas observou-se diferença entre as curvas de 0.026 , estatisticamente significativa.

Conclusão. Este trabalho apresenta uma sistematização do estudo elastográfico utilizando as informações obtidas durante a compressão e após a descompressão da amostra ultrassonográfica, demonstrando que a elastografia pode incrementar a avaliação do risco de malignidade para lesões caracterizadas pelo ultrassom.
\end{abstract}

Unitermos: Diagnóstico. Ultrassom. Mama. Elasticidade. Neoplasias da mama.

\section{INTRODUÇÃO}

A elastografia ultrassonográfica consiste de um software acoplado ao aparelho de ultrassom convencional que permite a avaliação dos diferentes tecidos presentes em uma amostra ultrassonográfica conforme a variação de sua compressibilida$\mathrm{de}^{1,2}$. Parte do princípio de que as lesões benignas são mais compressíveis do que as malignas na maioria das vezes e que este dado pode auxiliar para os diferentes diagnósticos de patologias clinicas, especialmente na mama por ser um órgão externo e pela sua consistência elástica ${ }^{3,4}$.
A técnica foi pioneiramente descrita por J onathan Ophir et al. ${ }^{5}$ durante a década de 1990 . Atualmente existem duas principais linhas de pesquisas para determinar sua aplicabilidade clínica. U ma baseada na avaliação das dimensões do nódulo antes e após compressões exercidas sobre a área de interesse utilizando um software que torna as lesões macias mais claras e as mais rígidas mais escuras ${ }^{6,7}$. A outra linha de pesquisa é baseada em um software que aplica diferentes espectros de cores aos tecidos conforme a sua rigidez, variando de vermelho para tecidos macios, verde para tecidos

1. Pós-graduando em medicina pela Faculdade de Ciências Médicas da Santa Casa São Paulo - Médico assistente do serviço de Diagnóstico por Imagem da Santa Casa de São Paulo, São Paulo, SP

2. Especialista em Diagnóstico por Imagem pelo CBR - Diretor do CTC-Genese, São Paulo, SP

3. Doutor em medicina pela Faculdade de Ciências Médicas da Santa Casa de São Paulo - Médico segundo assistente na Irmandade da Santa Casa de Misericórdia de São Paulo e Professor instrutor da Faculdade de Ciências Médicas da Santa Casa de São Paulo, São Paulo, SP

4. Doutor em medicina pela Faculdade de Ciências Médicas da Santa Casa de São Paulo - Primeiro assistente da Irmandade da Santa Casa de Misericórdia de São Paulo e Professor assistente da Faculdade de Ciências Médicas da Santa Casa de São Paulo, São Paulo, SP

5. Doutorado em medicina pela Faculdade de Ciências Médicas da Santa Casa de São Paulo - Professor titular da Faculdade de Ciências Médicas da Santa Casa de São Paulo, São Paulo, SP

6. Doutor em medicina pela Faculdade de Ciências Médicas da Santa Casa de São Paulo - Médico primeiro assistente da Santa Casa de Misericórdia de São Paulo e Professor instrutor da Faculdade de Ciências Médicas da Santa Casa de São Paulo, São Paulo, SP 
intermediários e azul escuro para tecidos rígidos ${ }^{8,9}$. Não se observa na literatura consenso quanto a melhor técnica ou classificação para a sua aplicação clínica, que tem como principais limitações a variação interobservadora descrita por alguns estudos e de como deve ser realizada a compressão da amostra durante o exame.

Este estudo propõe uma sistematização do exame elastográfico utilizando um software com variação de espectro de cores, com o objetivo de padronizar o exame e minimizar a variação interobservadora.

\section{Princípios físicos}

0 estudo elastográfico através do ultrassom consiste em uma extensão de uma das mais antigas ferramentas da medicina, a palpação, onde o médico avalia a forma e a rigidez do órgão em interesse $e^{2,3,5,6}$. A imagem elastográfica é gerada a partir da deformação (strain) dos tecidos que compõem uma amostra ultrassonográfica, produzindo ecos que são transformados em cores conforme a sua variação elástica, em que o vermelho corresponde a tecidos mais macios, o verde a intermediários e 0 azul escuro aos mais rígidos ${ }^{10,11,12}$.

A aplicação da deformação elástica no estudo ultrassonográfico é baseada em duas premissas: de que existem diferenças das propriedades mecânicas dos diversos tecidos componentes da mama e de que as informações obtidas por meio dos ecos formados pela força resultante são suficientes para detectar as diferenças por meio de estímulos mecânicos externos ou internos ${ }^{11}$.

As informações adquiridas são semelhantes às obtidas com a palpação manual, porém mais sensíveis e menos subjetivas. Como os tumores malignos geralmente são 5 a 10 vezes mais rígidos que o tecido mamário normal, a sua deformação será menor que a do parênquima mamário periférico, enquanto os tumores benignos tendem a sofrer maior variação elástica por serem mais compressíveis ${ }^{5,6}$.

Esta deformação promovida na amostra pode ser avaliada e comparada em dois momentos distintos, durante a compressão e após a descompressão (estresse) da área de interesse, em que a deformação (strain) resultante é diretamente proporcional ao estresse exercido, ou seja, quando se comprime um objeto ele tende a voltar a sua forma original após a descompressão $0^{13}$.

Como a força é resultante ao produto entre a massa e a aceleração, as imagens obtidas após a descompressão sofrem menor influência da forma em que a força é aplicada para 0 estudo. Isto se deve porque a força resultante é proporcional ao tempo de sua aplicação (aceleração) e é difícil de estabelecer um tempo padronizado para a compressão da amostra, que pode ser influenciado pelo tipo de parênquima e volume da mama $^{8}$. Durante a descompressão utiliza-se apenas a força resultante desta compressão que tende a ser homogênea se aplicada de forma sistematizada ${ }^{2,4}$.

\section{Métodos}

\section{Pacientes}

Estudo retrospectivo e prospectivo realizado no serviço de diagnóstico por imagem da Santa Casa de Misericórdia de São Paulo, sendo avaliadas as imagens obtidas de 245 pacientes com idade média de 45 anos (variação entre 24-70 anos) com
308 lesões encaminhadas para a realização de biópsia mamária percutânea ao Centro de Tomografia Computadorizada, CTC- Gênese, no período de 1 de maio de 2007 a 1 de março de 2008. Foram excluídas as primeiras 10 pacientes, cada uma com uma lesão, consideradas como curva de aprendizagem. As demais 235 pacientes apresentaram 298 lesões ao estudo ultrassonográfico.

Foi realizado inicialmente o estudo ultrassonográfico convencional (modo B) das lesões que foram classificadas conforme padronização proposta pelo léxico BI-RADS ${ }^{\circledR 14}$. Após 0 estudo convencional foi realizado o estudo elastográfico classificando as lesões conforme escores criados pelos autores, seguida pela biópsia percutânea realizada com agulha acoplada a pistola semiautomática de $14 \mathrm{G}$.

Tanto a classificação final do estudo convencional quanto a do estudo elastográfico foram comparadas com o resultado histológico. Foram calculadas sensibilidade, especificidade, acurácia diagnóstica e área dentro da curva ROC para os dois métodos ${ }^{15,16}$.

Para avaliação da concordância entre os observadores, foi realizado o teste Kappa seguindo os critérios de Landis \& Koch com o estudo retrospectivo de 115 nódulos realizado de forma aleatória. Todas as probabilidades de significância (valores de p) apresentadas são do tipo bilateral e valores menores que 0,05 considerados estatisticamente significantes. Para o cálcuIo, foi utilizado o software SAS 9.1.3 (Statistical Analysis System, Cary, NC, USA) ${ }^{17,18,19}$.

\section{Equipamento}

0 estudo convencional e a elastografia foram realizados por dois radiologistas, com 6 anos e 17 anos de experiência em imaginologia mamária cada, utilizando aparelho Ultrassonix Sonix-SP com sonda multifreqüencial de 5 a $14 \mathrm{mHz}$. Para 0 estudo da elastografia foi utilizado software especial para 0 aparelho da Ultrassonix, versão 3.0.2 (Beta1) cujos direitos de utilização experimental para pesquisa foram concedidos ao autor principal durante o período de estudo.

\section{Sistematização do estudo}

\section{Estudo ultrassonográfico convencional}

O estudo convencional deve ser precedido de palpação manual e de anamnese detalhada para avaliação global das mamas das pacientes. Deve ser realizado com as pacientes posicionadas em decúbito dorso-horizontal (DDH) para a maior parte das lesões ou em decúbito lateral-oblíquo para avaliação das lesões mais externas. 0 braço homolateral à mama estudada deve estar elevado sobre a cabeça $a^{7,8,9,20}$. Obtém-se imagens da área de interesse pelo modo $B$ por meio de varreduras nos eixos longitudinais e radiais ao mamilo, seguido pelo estudo Doppler colorido, em que se pode avaliar a vascularização dos nódulos seguindo os critérios estabelecidos pelo léxico BIRADS $^{\circledR 14}$. As medidas dos nódulos devem ser realizadas pelo modo B nos eixos crânio-caudal, látero-lateral e ântero-posterior abrangendo o maior eixo entre as duas margens externas. Para a documentação, deve-se armazenar as imagens do nódulo no quadro em que apresentar o seu maior eixo. 0 estudo convencional deve durar de 4 a 8 minutos. 
Fleury EFC et al.

\section{Estudo elastográfico}

A elastografia deve ser realizada com a paciente na mesma posição do exame convencional e com o transdutor orientado perpendicularmente à área de interesse. Antes de sua execução deve-se realizar compressões seriadas sobre a lesão para certificar o não-deslocamento lateral da mesma. Após ativar a elastografia, deve-se realizar uma compressão manual de forma contínua sobre a mama da paciente na área a ser estudada, perpendicular ao peitoral, até encontrar resistência não forçada do parênquima. Quando encontrada esta resistência deve-se relaxar a mão proporcionando a descompressão espontânea da mama. A área de estudo selecionada deve incluir desde o tecido celular subcutâneo até o músculo peitoral e também a periferia do nódulo por até $0,5 \mathrm{~cm}$ (Figura 1 ).

As imagens obtidas devem ser avaliadas em tempo real e por meio do cinememory, em que pode-se avaliar de forma retrospectiva o comportamento do nódulo durante a compressão e após a descompressão da amostra. Deve-se armazenar para a documentação fotográfica um quadro do nódulo durante a compressão e outro após a descompressão. Pode-se ainda gravar o filme do estudo elastográfico em mídia digital. 0 tempo de estudo deve variar entre 30 segundos e dois minutos por nódulo. A documentação deve ser realizada de forma que qualquer profissional apto à realização do exame possa reproduzir os resultados encontrados pelo executor do estudo.

\section{Classificação elastográfica}

A classificação elastográfica proposta inclui quatro escores conforme a variação de cor durante a compressão e após a descompressão da área de interesse. 0 escore 1 é dado às lesões que apresentam o mesmo espectro de cores do tecido mamário periférico durante o estudo. 0 escore 2 é conferido às lesões que após a descompressão apresentam variação de cor para mais macia abrangendo mais que $50 \%$ da amplitude do nódulo quando comparada com a imagem durante a compressão. Já o escore 3 é dado às lesões que após a descompressão apresentam variação de cor em menos de $50 \%$ da área do nódulo (entre $10 \%$ e $50 \%$ ). Por fim, no escore 4 encontramos as lesões que não apresentam variação significativa na cor durante a compressão e após a descompressão do parênquima, permanecendo azul nas duas imagens (Figura 2).

\section{Resultados}

Os valores preditivos positivos, negativos, sensibilidade, especificidade e acurácia diagnóstica dos scores da elastografia obtidos foram os seguintes: $79.25 \%, 97.10 \%, 85.71 \%, 95.51 \%$ e $93.88 \%$ (VP: 44/298; VN: 236/298; FP: 11/298; FN: 7/298), e para a ultrassonografia de $43.43 \%, 96.92 \%, 87.76 \%, 77.14 \%$ e 78.91\% (VP: 45/298; VN: 190/298; FP: 57/298; FN: 6/298).

A área dentro da curva para a elastografia foi de $0.952 \mathrm{com}$ intervalo de confidência entre 0.910 e 0.966 , erro de 0.023 , e de 0.867 com intervalo de confidência entre 0.823 e 0.903 , erro de 0.0333 para o ultrassom. Quando comparadas as áreas, observou-se diferença entre as áreas dentro das curvas de 0.026 , estatisticamente significativa (Figura 3). Ao nível de significância de $5 \%$, observou-se um grau de concordância quase perfeito entre os observadores (Weighted Kappa $=0.847$, erro de 0.047 e $p<0.0005$ ).
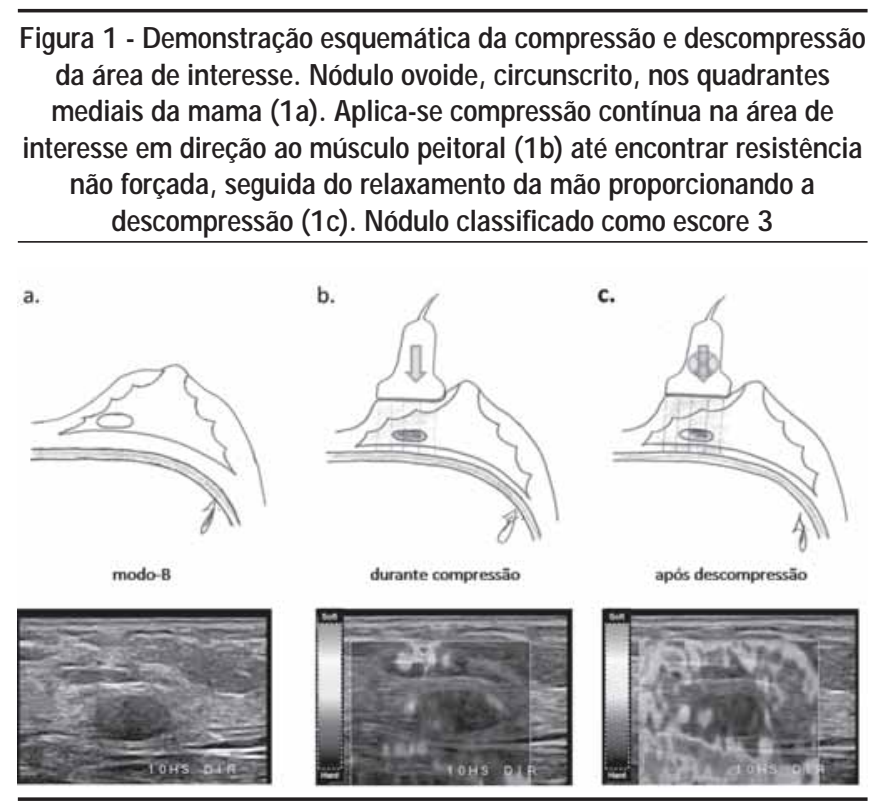

Figura 2 - Classificação proposta dos nódulos mamários durante a compressão e após a descompressão da mama. Escore 1 representa nódulo que tem o mesmo comportamento do tecido mamário periférico. Escore 2 apresenta variação de cor para mais macia ocupando mais de $\mathbf{5 0} \%$ do nódulo após a descompressão. Escore 3 tem variação em menos de $50 \%$ do nódulo ( 10 a $50 \%$ ). Escore 4 sem variação significativa

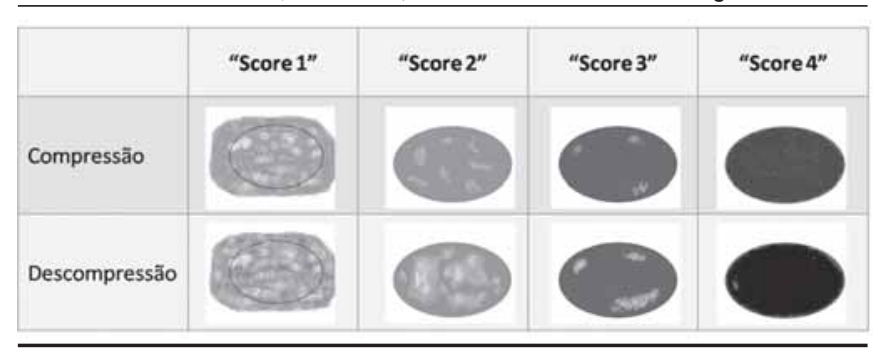

Figura 3 - Curvas ROC para a elastografia e modo B. Há incremento para detecção de malignidade utlizando a elastografia $(p=0.026)$

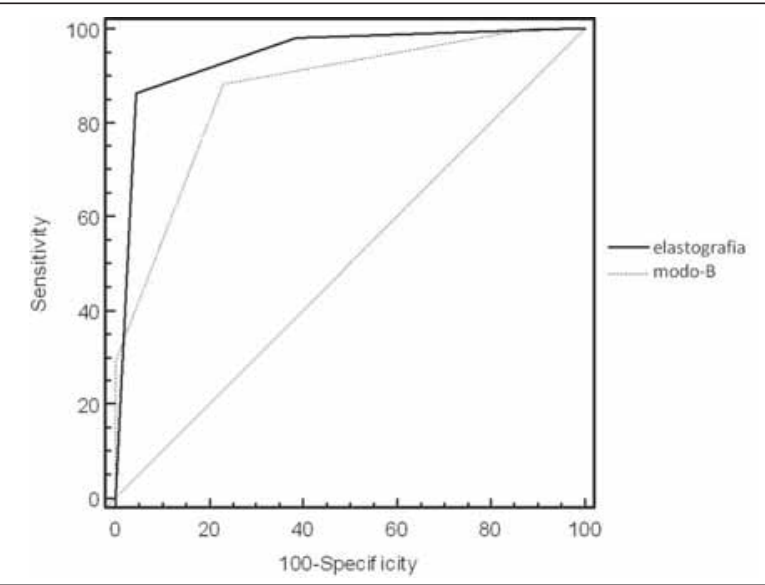




\begin{tabular}{|c|c|c|c|c|c|}
\hline Estudo & Casos & Obervador & Compressão & Acurácia diagnóstica & Classificação \\
\hline Moon et al. & 100 & 1 & $0,4-0,6 \mathrm{~cm}$ & $87,0 \%$ & $\begin{array}{l}\text { Segmentação das imagens por meio do brilho avaliando margem, } \\
\text { área, distância e solidez }\end{array}$ \\
\hline Giuseppetti et al. & 91 & 2 & Gradual & $82,4 \%$ & $\begin{array}{l}\text { Variação de cores para discriminar lesões benignas de malignas } \\
\text { (Cinco escores) }\end{array}$ \\
\hline Regner et al. & 89 & 5 & Gradual & - & $\begin{array}{l}\text { Varição da área, diâmetros ântero-posterior e laterais, e suas } \\
\text { relações (variação interobservadora) }\end{array}$ \\
\hline Ito et al. & 111 & 1 & Seriada & $88,3 \%$ & $\begin{array}{l}\text { Variação de cores para discriminar lesões benignas de malignas } \\
\text { (Cinco escores semelhantes ao Giuseppetti) }\end{array}$ \\
\hline Burnside et al. & 98 & 3 & Ciclíca em 10\% da & $90,3 \%$ & $\begin{array}{l}\text { Persistência das imagens do modo-B durante a elastografia associado } \\
\text { a avaliação do ruído (Cinco escores distintos dos anteriores) }\end{array}$ \\
\hline Fleury et al. & 298 & 2 & Gradual/ Descompressão & $94,0 \%$ & $\begin{array}{l}\text { Variação de cores comparando imagens durante a compressão e } \\
\text { após a descompressão por meio de quatro escores }\end{array}$ \\
\hline
\end{tabular}

\section{Discussão}

Há mais de uma década os métodos de elastografia vêm ganhando atenção para o estudo de tecidos moles por meio do ultrassom com a perspectiva clínica de poder detectar precocemente lesões que determinem alterações patológicas nos teci$\operatorname{dos}^{1,2,3,4,5}$. Pode ser utilizada como ferramenta complementar ao estudo ultrassonográfico convencional e contribuir para a orientação da conduta adotada $6,7,8,9$, principalmente nos nódulos indeterminados.

0 estudo pioneiro realizado em 1991 por Ophir et al. ${ }^{5}$ propunha classificar os nódulos segundo a variação elástica, partindo do princípio que lesões benignas eram mais moles enquanto a maioria das malignas eram mais rígidas. A partir de então, vários trabalhos vêm sendo publicados, porém sem apresentar consenso quanto a padronização da técnica ou classificação a ser empregada, a maioria utilizando apenas a comparação de imagens pré e após a compressão da área de interesse ${ }^{6,7,8,9,20}$ (Tabela 1).

Parece não haver consenso também quanto ao melhor software a ser utilizado. Os estudos que utilizam o software para avaliar a alteração das dimensões dos nódulos durante a compressão baseiam-se na variação do brilho de claro para 0 escuro, demonstrando que as margens das lesões malignas tendem ficar mais evidentes durante o período de compressão do que nos nódulos benignos, achado provavelmente relacionado a desmoplasia encontrada nos carcinomas ${ }^{6,7,20 .}$. Para 0 estudo utiliza-se a área do nódulo ou as medidas nos maiores eixos antes e durante a compressão. A maioria dos artigos apresenta classificações próprias, algumas mais complexas associando as alterações de brilho nos nódulos com as alterações das medidas obtidas ${ }^{20}$. U ma das limitações relatadas é a variação interobservadora para determinados observadores e a baixa especificidade para outros ${ }^{7}$.

Já os ensaios utilizando os softwares que aplicam variação do espectro de cores apresentam resultados animadores, porém sem o estudo da variação interobservadora ${ }^{8,9}$. N ão apresentam consenso quanto a melhor técnica para a realização do estudo ou quanto a melhor classificação a ser utilizada. Itho et al. ${ }^{8}$ propõem o estudo por meio de compressões contínuas sobre 0 parênquima mamário, avaliando as imagens obtidas no período precoce da compressão por acreditar que sofrem menor variação em relação aos outros períodos. Apresentam uma classificação composta por 5 escores sendo os escores 1,2 e 3 benignos, e os 4 e 5 suspeitos para malignidade baseados em critérios esquemáticos de variação de cor.

Como revisado, não se observa um consenso quanto a melhor forma de aplicação da força de compressão e da sistematização do estudo, tampouco de como deve ser feita a sua documentação. Não há relatos da valorização das imagens após a descompressão, sendo valorizadas apenas as imagens pré e após a compressão da área de interesse. Acreditamos que realizando o estudo durante a compressão e após a descompressão, com as compressões realizadas conforme a sistematização proposta, pode-se não só padronizar o estudo como criar parâmetros comparativos entre estes dois momentos. Referente a dificuldade encontrada em quantificar a força a ser aplicada para a compressão do parênquima, acreditamos que possa ser vencida utilizando esta forma de compressão gradual, até encontrar a resistência da mama não forçada, em que há maior valorização do momento de descompressão.

Quanto à avaliação das margens, acreditamos não ser um bom parâmetro para a diferenciação das lesões benignas de malignas, principalmente por não possibilitar uma definição das mesmas durante o exame, já que se trata de um estudo dinâmico o que interfere na sua avaliação. Este pode ser um dos fatores que contribuiu para a variação interobservadora ${ }^{7}$ de estudos anteriores e um fator limitante para a sua documentação e reprodução. Acreditamos que a utilização do software baseado em espectro de cores apresenta maior aplicabilidade clinica por ser de interpretação menos complexa, baseada apenas na avaliação visual da variação de cores.

Acreditamos ainda que quanto mais simples e objetiva a classificação, mais fácil a sua implementação na prática clínica. Devido à elastografia ser uma ferramenta complementar ao estudo ultrassonográfico ela não irá determinar de forma isolada a conduta frente a uma lesão, irá auxiliar a indicar uma biópsia ou o seu seguimento. Os escores criados pelos autores foram baseados no estudo dinâmico da ressonância magnética (RM) amplamente utilizado na prática clínica, no qual se observam três padrões de curva cinética da vascularização: a curva I característica de lesões benignas, a II de lesões indeterminadas e a III de lesões suspeitas. Estas curvas são utilizadas como 
dados complementares as imagens de alta resolução espacial e auxiliam para a diferenciação de nódulos benignos de malignos. São consagradas por proporcionar uma avaliação simples e de fácil reprodutibilidade ${ }^{21,22}$.

Os escores da elastografia propostos seguem padrão semeIhante, onde 0 escore 1 são lesões formadas não reais, como 0 lóbulo de gordura, o escore 2 lesões benignas, o escore 3 lesões indeterminadas, porém com baixa probabilidade de malignidade (VPN de 93,41\% em nossa série) e o escore 4 são lesões suspeitas para malignidade. Como os radiologistas e os mastologistas já estão acostumados com as curvas cinéticas da RM acreditamos que teriam pouca dificuldade para se adaptar a esta classificação.

Por fim, a padronização da documentação, pouco valorizada em outros ensaios, deve ser de fundamental importância, por ser a forma de comunicação do executor do exame com o paciente e o médico solicitante. Pode ser fotográfica ou em mídia digital, e deve possibilitar que qualquer outro profissional apto reproduza os achados encontrados no exame de forma visual.

A elastografia é um método promissor para auxiliar no diagnóstico de lesões mamárias, porém ainda sem nenhuma padronização técnica e de documentação. Acreditamos que por meio desta sistematização proposta poderemos padronizar o estudo, facilitando a sua aplicabilidade clínica e sua reprodutibilidade.

\section{Conflito de interesse: não há}

\section{SUMmarY}

Proposal for the systematization of the elastographic study OF MAMMARY LESIONS THROUGH ULTRASOUND SCAN

Oвjective. Proposal of systematization for the elastographic study in the ultrasound routine.

METHODS. Evaluation was made of 308 patients forwarded to the breast intervention service in the CTC-Genesis from May 1 , 2007 to March 1, 2008 to perform percutaneous breast biopsy. Prior to the percutaneous biopsy, an ultrasound study and an elastography were performed. Lesions were primarily analyzed and classified according to the Bi-Rads ${ }^{\circledR}$ lexicon criteria by the conventional ultrasound scan (B mode). The elastography was then performed and analyzed in accordance with the systematization proposed by the authors, using images obtained during compression and after decompression of the area of interest. Lesions were classified following the system developed by the authors using a four-point scale, where scores (1) and (2) were considered benign, score (3) probably benign and score (4) suspicion of malignancy. Results obtained by the two methods were compared with the histological results using the areas within the ROC (receiver operator curves) curves.

RESULTS. The area within the curve for elastography was of 0.952 with a confidence interval between 0.910 and 0.966 , error of 0.023 , and of 0.867 with a confidence interval between 0.823 and 0.903 , error of 0.0333 for the ultrasound. When the areas were compared, a difference between the curves of 0.026 was observed, which was statistically significant.

Conclusion. This work shows the systematization of the elastographic study using information obtained during compression and after decompression of the ultrasound scan sample, thus showing that elastography might enhance the assessment of risk of malignancy for lesions characterized by the ultrasound. [Rev Assoc Med Bras 2009; 55(2): 192-6]

KEY WORDS: Diagnosis. Ultrasound. Breast. Elasticity. Breast Neoplasms.

\section{ReferênCIAS}

1. Hoyt K, Forsberg F, Ophir J. Analysis of a hybrid spectral strain estimation technique in elastography. Phys Med Biol. 2006;51(2):197-209.

2. O'Donnell M, Skovoroda AR, Shapo BM, Emelianov SY. Internal displacement and strain imaging using ultrasonic speckle tracking IEEE Trans. Ultrason Ferroelectr Freq Control. 1994;41(3):314-25.

3. Emelianov SY, Lubinski MA, Weitzel WF, Wiggins RC, Skovoroda AR, O'Donnell M. Elasticity imaging for early detection of renal pathology. Ultrasound Med. Biol. 1995;21(7):871-83.

4. Krouskop TA, WheelerF, Kallel F, Garra B, Hall T. The elastic moduli of breast and prostate. tissues under compression. Ultrason Imaging. 1998;20(1):151-9.

5. Ophir J, Cespedes I, Ponnekanti H, Yazdi Y, Li X. Elastography: a quantitative method for imaging the elasticity of biological tissues. Ultrason Imaging 1991;13(2):111-34.

6. Moon WK, Chang RF, Chen CJ, Chen DR, Chen WL. Solid breast masses: classification with computer-aided analysis of continuous US images obtained with probe compression. Radiology. 2005;236(2):458-464.

7. Regner DM, Hesley GK, Hangiandreou NJ. Breast lesions: evaluation with US strain imaging-clinical experience of multiple observers. Radiology. $2006 ; 238(2): 425-37$

8. Itoh A, Ueno E, Tohno E. Breast disease: clinical application of US elastography for diagnosis. Radiology. 2006;239(2):341-50.

9. Giuseppetti GM, Martegani A, Di Cioccio B, Baldassarre S. Elastosonography in the diagnosis of the nodular breast lesions: preliminary report. Radiol Med (Torino). 2005;110(1):69-76.

10. Hall TJ, Zhu Y, Spalding CS. In vivo real-time freehand palpation imaging. Ultrasound Med Biol. 2003;29(4):427-35.

11. Konofagou EE, Ophir J, Krouskop TA, Garra BS. Elastography: from theory to clinical applications; 2003. In: Summer Bioengineering Conference, J une 25-29. Key Biscayne, Florida.

12. Hall TJ . AAPM/RSNA physics tutorial for residents: topics in US: beyond the basics: elasticity imaging with US. Radiographics. 2003:23(6):1657-71.

13. Hooke's Law. Encyclopedia B ritannica. [cited 2008 mar 1]. Available from: http://www. britannica.com/eb/article-9040985.

14. American College of Radiology. Breast imaging reporting and data system (BI-RADS), ultrasound. 4th ed. Reston: American College of Radiology; 2003. [cited 2007 mar 30]. Available from: http://www.acr.org/ SecondaryM ainM enuCategories/quality safety/BIRADSAtlas/ BIRADSAtlasexcerptedtext/BIRADSU ItrasoundFirstEdition/ACRBIRADSU SLexiconClassificationFormDoc1.aspx.

15. Gonen M. Analyzing receiver operating characteristic curves using SAS. Cary: SAS Press; 2007.

16. Hosmer DW, Lemeshow S. Applied logistic regression. $2^{\text {nd }}$ ed. New York: Wiley-Interscience; 2000. p.20-46.

17. Cohen J. Weighted kappa: nominal scale agreement with provision for scaled disagreement or partial credit. Psychol Bull. 1968;70(2):213-20.

18. Landis J R, Kock GG. The measurement of observer agreement for categorical data. Biometrics. 1977;33(1):159-75.

19. SAS Institute. SAS 9.1.3 help and documentation. Cary: SAS Institute; 2000-2004.

20. Burnside ES, Hall TJ, Sommer AM, Hesley GK, Sisney GA, Svensson WE, et al. Differentiating benign from malignant solid breast masses with US strain imaging. Radiology. 2007;245(4):401-10.

21. Kuhl C. The current status of breast mr imaging: part I. Choice of technique, image interpretation, diagnostic accuracy, and transfer to clinical practice. Radiology. 2007;244(4):356-78.

22. Macura KJ, Ouwerkerk R, Jacobs MA, Bluemke DA. Patterns of enhancement on breast MR images: interpretation and imaging pitfalls. Radiographics. 2006;26(7):1719-34.

Artigo recebido: 25/03/08

Aceito para publicação: 21/07/08 